\title{
A Robust Lane Detection Method for Urban Roads
}

\author{
Mohamed Alaa*, Gerges M. Salama, Ahmed I. Galal, Hesham F. A. Hamed \\ Department of Electrical Engineering, Faculty of Engineering, Minia University, Minia, Egypt \\ *Corresponding author E-mail: mohamed.alaa@mu.edu.eg
}

\begin{abstract}
Many intelligent transportation systems, such as advanced driver assistance systems (ADAS), have been being developed to increase traffic safety. ADAS have the potential to save lives and reduce crashes by eliminating the human error in the driving process. The Lane detection system is designed to identify and estimate the position of lane boundaries in front of the ego-vehicle. Thus, it serves as a crucial and fundamental component in various ADAS, for instance, lane keeping assistance and lane departure warning assistance systems. In this paper, a proposed method of visionbased lane detection in urban roads is presented. First, we define a region of interest to exclude the misleading parts of the road image, then a bird's-eye view of the road in front of the ego-vehicle is obtained by employing the inverse perspective mapping. Second, we utilize the distinct colors of lane markings to achieve robust lane markings candidate detection. Finally, the estimated lane boundaries are represented by quadratic models whose parameters are estimated from the detected lane pixels using the RANSAC algorithm. Furthermore, we present a thorough evaluation of the detection performance of the proposed method using the ground-truth data of the Caltech dataset and a comparative analysis between the quadratic model used in the proposed method and other models presented in the literature. Detection results show the effectiveness of the proposed method in detecting lane boundaries in different conditions in urban roads, including curved lanes, shadows, illumination variations and presence of street writings. Moreover, the overall process takes an average time of 30.63 milliseconds per frame.
\end{abstract}

Keywords: Lane detection, advanced driver assistance systems, Caltech dataset, bird's-eye view transformation, region of interest, RANSAC.

\section{INTRODUCTION}

Road traffic accidents are more and more being perceived as one of the most serious public health issues. Thousands of people are killed and injured on roads all over the world every day $[1,2]$. Automotive passive safety systems, such as seat belts and air bags, were invented to minimize the damages resulted from the road accidents [3]. However, the efficiency of this type of safety systems is limited as they try to reduce the consequences of

Received :27Ougest,2020 Accepted :14Septamber,2020 vehicle crashes rather than preventing the crashes from ever happening.

The advancements occurred in the automotive technologies, have led to the development of intelligent safety systems called the advanced driver assistance systems (ADAS). The main target of ADAS is to eliminate the human

error in the driving process; consequently, this helps prevent or reduce the likelihood of crash occurrences. Through the perception of the surrounding environment of a vehicle via multiple sensors like Radars, Lidars, Ultrasonic 13 
sensors and cameras, ADAS are able to deliver assistance to the drivers while driving and warn them about risky situations that may lead to traffic accident [4] .

Due to the advances recently made in camera technologies, vision-based ADAS have been and still an active research area because they have the potential to dramatically decrease the number of traffic accidents [4]. The lane detection task provides information which helps locate the lane boundaries on the road in front of the ego-vehicle. Thus, it's a crucial and fundamental component of various vision-based ADAS including lane departure warning assistance (LDWA) [5] and lane keeping assistance (LKA) [6]. They are utilized to prevent unintended departures of the ego-lane so that the vehicle can navigate safely within it with no sudden deviations that may result in a crash.

Various lane detection methods were presented in the literature. [7] reduced the shadows casted by trees by filtering edges that weren't vertical and aligned with the edges resulted from lane boundaries. [8] used the illumination invariance to extract white and yellow lane boundaries. This method has a good detection performance for night as well as lighting changes. Yet, for the case that the lane boundary is worn or blurred, the detection performance is decreased. [9-12] proposed several lane models ranging from simple models as straight lines to more complex models such as flexible splines. The hough transform was employed to extract lines from the road images in [5]. Then, the lane models were fitted to these lines.

The lane detection methods were often applied to the road images to localize the lanes by extracting low-level features. These features could be color [13], texture [14] and the gradient intensity [15]. Accordingly, they were used to extract the lane-like pixels from the road area in the image. In [13], the color clustering method was used for lane bounda- ries detection. This method converted the original image from the RGB color model into the Lab color model, then employed the clustering algorithm to extract the lane-like pixels in the Lab color space. The width feature information of lane boundaries as well as filtering were used to detect the lane boundaries in [16]. Authors in [17] utilized the gradient information of lane edges to perform the lane detection. They employed the adaptive Canny edge detection to maximize the edge intensities of the lane-like pixels because it can dramatically reduce the impact of noisy edges and adapt to several harsh road conditions. However, this method is widely affected by the changes in lighting situations.

Most of the existing lane detection methods were designed for highways with almos straight and quite clear lane boundaries. However, this task is increasingly difficult in urban roads where some challenges rise. For instance, street writings, curved lane boundaries, shadows casted by trees, occlusion by moving vehicles and illumination variations in driving scenes.

In this paper, we propose a lane detection method whose performance is stable under diverse illumination conditions, and it exhibits robustness against the varying driving scenes in urban roads. In addition, we present a thorough evaluation of the detection performance of proposed method using the ground-truth lane points of the Caltech dataset. Moreover, a comparative analysis of the detection performance and the computational time is conducted between the quadratic model utilized in the proposed method and other models adopted in the literature.

The rest of this paper is organized as follows: In section 2, we present a description of the proposed lane detection method. Section 3, provides the simulation results as well as quantitative evaluation of the detection performance of the proposed method and com- 
parative analysis, followed by a discussion in section 4. Lastly, conclusions are provided in section 5 .

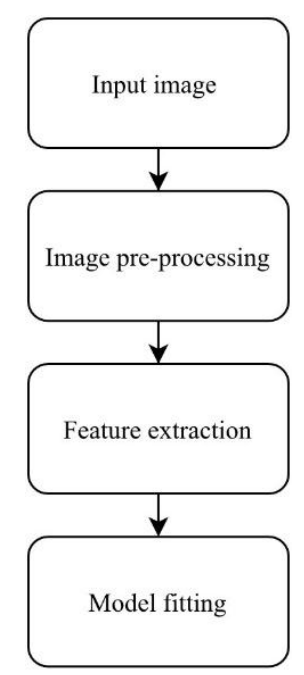

Fig. 1. The proposed method block diagram.

\section{PROPOSED METHOD}

The proposed method consists of three sub methods that operate sequentially: image pre-processing, feature extraction and model fitting as shown in Fig. 1.

\section{A. Image pre-processing}

The main target of image pre-processing is to facilitate the concentration on the features of interest in the road images for the following sub methods. First, a region of interest (ROI) was defined and applied to the input image. It helped focus on the relevant parts and exclude the misleading parts of the image, which may confuse the feature extraction sub method. In the camera perspective, lane boundaries tend to converge at the horizon. Furthermore, they don't appear parallel to each other as in reality. This type of distortion is called the perspective distortion. That's why the inverse perspective mapping (IPM) was employed to obtain the bird's-eye view (BEV) which was a top-down view of the road in front of the egovehicle [12]. To get the BEV of the input road image, we assumed a flat road, then the camera intrinsic (focal length and optical center) and extrinsic (pitch angle, yaw angle, and

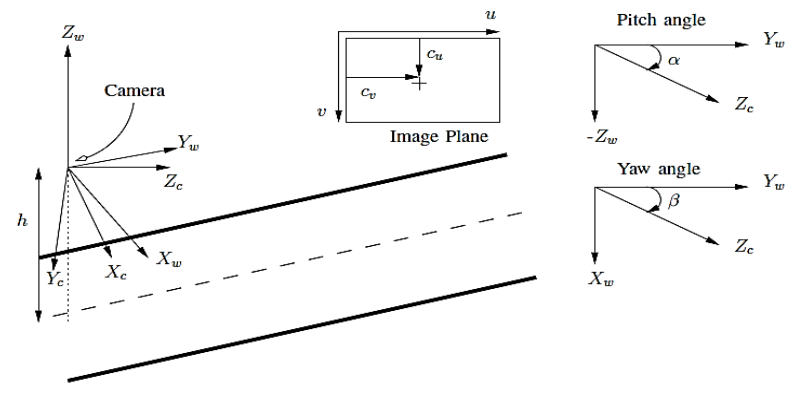

Fig. 2. Left: the coordinate axes (world, camera, and image frames). Right: definition of pitch $\alpha$ and yaw $\beta$ angles [9].

height above ground) parameters were used to perform the IPM transformation. There existed a set of different coordinate axes as illustrated in Fig. 2. A world frame $F_{w}=$ $\left\{X_{w}, Y_{w}, Z_{w}\right\}$ was defined and centered at the camera optical center. A camera frame $F_{c}=\left\{X_{c}, Y_{c}, Z_{c}\right\}$, and an image frame $F_{i}=$ $\{u, v\}$ were also defind as shown in Fig. 2. We assumed that the camera frame $X_{c}$ axis stayed in the world frame $X_{w} Y_{w}$ plane. A pitch angle $\alpha$ and yaw angle $\beta$ were allowed for the optical axis but no roll. The projection of any point in the image plane $P_{i}=\{u, v, 1,1\}$ on the road plane, can be found by applying the homogeneous transformation $\mathrm{H}$ :

$$
H=h\left[\begin{array}{cccc}
\frac{-1}{f_{u}} c_{2} & \frac{1}{f_{v}} s_{1} s_{2} & \frac{1}{f_{u}} c_{u} c_{2}-\frac{1}{f_{v}} c_{v} s_{1} s_{2}-c_{1} s_{2} & 0 \\
\frac{1}{f_{u}} s_{2} & \frac{1}{f_{v}} s_{1} c_{1} & \frac{-1}{f_{u}} c_{u} s_{2}-\frac{1}{f_{v}} c_{v} s_{1} c_{2}-c_{1} c_{2} & 0 \\
0 & \frac{1}{f_{v}} c_{1} & \frac{-1}{f_{v}} c_{v} c_{1}+s_{1} & 0 \\
0 & \frac{-1}{h f_{v}} c_{1} & \frac{1}{h f_{v}} c_{v} c_{1}-\frac{1}{h} s_{1} & 0
\end{array}\right]
$$

where $h$ is the height of the camera frame above the ground plane, $\left\{f_{u}, f_{v}\right\}$ are the horizontal and vertical focal lengths, respectively, $\left\{c_{u}, c_{v}\right\}$ are the coordinates of the optical center, and $c_{1}=\cos \alpha, c_{2}=\cos \beta, s_{1}=$ $\sin \alpha, s_{2}=\sin \beta$. This transformation can 
be efficiently computed in the matrix form for hundreds of points as the following :

$$
P_{g}=H \cdot P_{i}
$$

where $P_{g}$ is the point on the ground plane corresponding to the point $P_{i}$ on the image plane. Furthermore, this process can be inverted to project a point from the ground plane into the image plane. Fig. 3 illustrates the original road image taken by a front-facing camera mounted on the ego-vehicle with the ROI in brown and the corresponding generated $\mathrm{BEV}$ image. As can be seen, the lane boundaries have fixed width and appear parallel to each other in the BEV image.

\section{B. Feature extraction}

This sub method is concerned with detecting and extracting the features of interest for the model fitting sub method. It produces a black and white image where the candidate lane pixels are represented by the white pixels. There are various techniques for detecting lane markings. Color-based techniques rely on the fact that lane markings have pre-defined colors (e.g., white or yellow) as in [8]. Other techniques utilize the gradient intensity information since lane markings tend to have obvious appearance relative to the road texture [17]. In the proposed method, we adopted the lane detector presented in [18]. For the purpose of detecting lane markings, the BEV image was initially converted to the grayscale intensity image upon which the lane detector was applied. Assuming that lane-like pixels were

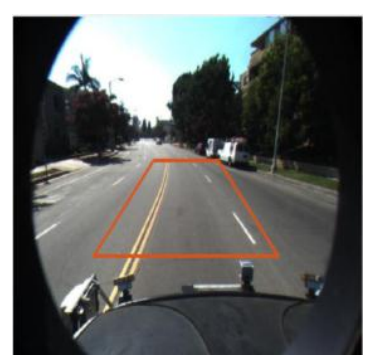

(a)

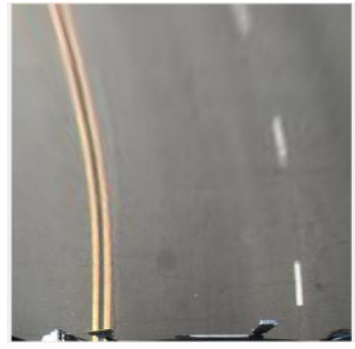

(b)

Fig. 3. (a) Original road image with ROI. (b) The corresponding BEV image.

the pixels with higher intensity values compared to neighboring pixels on either side in the BEV image represented in the grayscale intensity, the detection was consequently carried out by independently filtering each row of the image, depicted as $\left\{x_{i}\right\}_{i=1}^{W}$. This resulted in a new filtered row data array, denoted as $\left\{y_{i}\right\}_{i=1}^{W}$ that could be obtained as the following :

$y_{i}=2 x_{i}-\left(x_{i-l}+x_{i+l}\right)-\left|x_{i-l}-x_{i+l}\right|$

and this process was repeated for each row in the image. This filter produced high responses for positions with $x_{i}$ values that were higher than those of their neighbors on the left and right at a distance. We set up $l$ to 25 centimeters in vehicle coordinates to detect candidate lane pixels that were 25 centimeters in width, which was the approximate width of lane markings, in order to reject outliers that could be wider. In (3), the last term penalized cases in which the difference between the left and right neighbors was high, so that a higher response was given to positions with similar left and right neighbors. The BEV image and the generated black and white image are shown in Fig. 4.

\section{Model fitting}

After extracting the lane features in the previous sub method, $\{x, y\}$ coordinates of the detected lane pixels, estimated lane boundaries

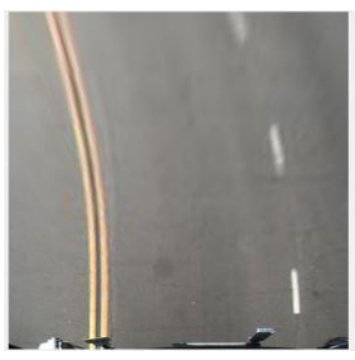

(a)

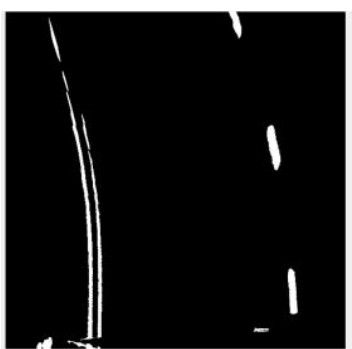

(b) 
Fig. 4. (a) BEV image. (b) The corresponding black and white image.

were modeled through fitting mathematical models ( functions) to the candidate lane pixels. Transforming the image into the BEV simplifies the fitting process of the mathematical models since the lane boundaries become similar in curvature and the road's width is roughly constant. Model fitting is effective in this context. Moreover, it is sufficient and well-suited for the task of obtaining compact high-level representations of lane booundaries without the need for more sophisticated methods that introduce extra computations which may result in not conforming the computational time requirements.

Several mathematical models were introduced in the literature.Authors in [9] utilized the linear model for modeling lane boundaries, whereas the quadratic model was used by [10]. Cubic model [11] and spline models [12] were also introduced in the literature. The quadratic model was used in the proposed method because it has the potential to model curved lane boundaries. Thus, it's more generic and not confined to straight roads, as in the case of linear model. Furthermore, it exhibits more computational efficiency than higherorder models, such as cubic models and splines, on account of the fact that estimating more parameters demands extra computations. In regards to the model fitting process, random sample consensus (RANSAC) algorithm [19] was used to estimate quadratic model parameters of the estimated lane boundaries from the previously detected lane points. It's a robust fitting algorithm that uses the leastsquares error (LSE) technique iteratively to fit a mathematical model to data points. Its iterative process gives it the ability to reject outliers and noise that may result from the feature extraction sub method, due to blurred lane markings, tree shadows and flawed road surfaces. In our proposed method, RANSAC was given the values of $x$ and $y$ where $\{x, y\}$ de- picts the row and column positions of the detected lane pixels, and $a, b, c$

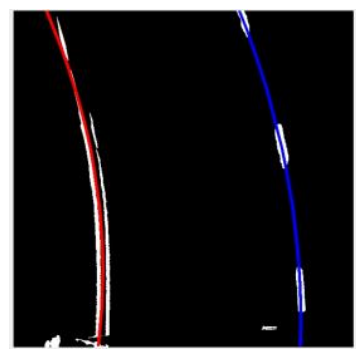

(a)

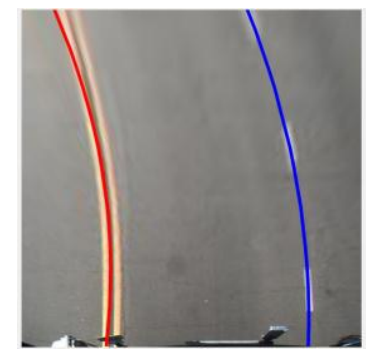

(b)
Fig. 5. (a) Black and white image with overlaid estimated lanes. (b) The corresponding BEV image with overlaid estimated lanes.

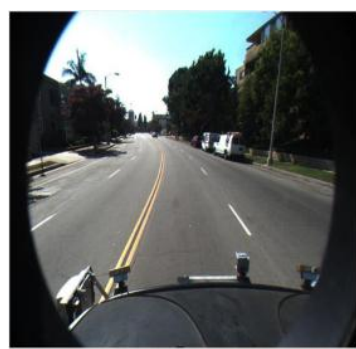

(a)

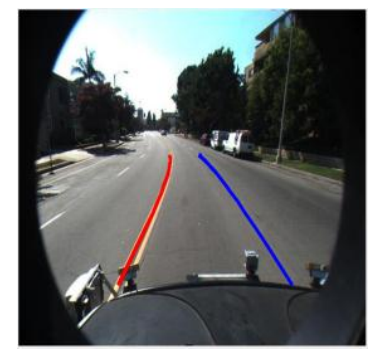

(b)
Fig. 6. (a) Original road image. (b) The corresponding lane-detected image.

are the quadratic model parameters estimated by RANSAC in (4) :

$$
y=a x^{2}+b x+c
$$

For the purpose of estimating the parameters of the quadratic model, the best fitting quadratic model should have the (LSE) according to the following :

$$
\begin{aligned}
& L S E=\sum_{i=1}^{n}\left[y_{i}-y_{e}\right]^{2} \\
& L S E=\sum_{i=1}^{n}\left[y_{i}-\left(a x_{i}^{2}+b x_{i}+c\right)\right]^{2}
\end{aligned}
$$


where $n$ is the number of the potential lane points, $\left(x_{i}, y_{i}\right)$ is the actual $x$ and $y$ coordinates of the $i$-th potential lane pixel and $y_{e}$ is the estimated $y$ coordinate. Since we were trying to find out the parameters that would give the minimum LSE, the partial derivatives of the error equation with respect to $a, b$, and $c$, were calculated. With setting those partial derivatives to zero, we got :

$$
\begin{aligned}
& \frac{\partial L S E}{\partial a}=2 \sum_{i=1}^{n} x_{i}^{2}\left[y_{i}-\left(a x_{i}^{2}+b x_{i}+c\right)\right]=0(7) \\
& \frac{\partial L S E}{\partial b}=2 \sum_{i=1}^{n} x_{i}\left[y_{i}-\left(a x_{i}^{2}+b x_{i}+c\right)\right]=0(8) \\
& \frac{\partial L S E}{\partial c}=2 \sum_{i=1}^{n}\left[y_{i}-\left(a x_{i}^{2}+b x_{i}+c\right)\right]=0
\end{aligned}
$$

By simplifying the three partial derivative equations above and arranging them in the matrix form $A X=B$, the solution to this equationwhich was the values of $a, b$, and $c$ could be found using lower-upper (LU) decomposition. After these values were found, the quadratic models were obtained and overlaid on the image to highlight the lane boundary. It's worth pointing out that this process had to be carried out once for the right boundary and once for the left one. Fig. 5 shows the estimated lane boundaries Overlaid on the black and white image and on its corresponding BEV image. Additionally, Fig. 6 illustrates the original road image representing the input to the proposed method, and the corresponding lane-detected image which represents the output of the proposed method.

\section{SIMULATION RESULTS}

The proposed method has been prototyped using MATLAB running on Linux Ubuntu on a PC with Intel(R) Corei7-6500 CPU @ 2.50 $\mathrm{GHz}$ and $8.00 \mathrm{~GB}$ RAM.

\section{A. Performance Evaluation}

The interest in developing vision-based ADAS, has been growing for many years. The performance evaluation for these systems has become a crucial task to check the accuracy of their performances and investigate whether the results meet predefined safety requirements or not.

The performance evaluation can be conducted by comparing our lane detections to ground-truth data, and measuring the amount of matching between them. This provides us with a characterization of the proposed method performance both visually and quantitatively. Thus, we can figure out where and when it may fail to refine and improve it. Accordingly, we used the Caltech lanes dataset published in [12]. It consists of four video sequences (Cordova1, Cordova2, Washington1, Washington2) with 250, 406, 337, 232 frames, respectively. They exhibit different challenging scenarios, including curved lane boundaries, writings on the street and shadows. The authors of this dataset has hand-labeled all visible lanes in the video frames. Fig. 7 shows examples of the Caltech dataset frames. Likewise, the ground-truth lane points represented in image coordinates for one of the frames, can bee seen in Fig. 8.

The proposed method was applied on each frame of the four video sequences forming the Caltech dataset. As can be seen from Fig. 9, the proposed method accurately detects lane boundaries, even though there are vehicles passing close to them. Furthermore, it can detect the lane boundaries in complex cases such as when curved, when affected by shadows and in presence of street writings. As mentioned previously, ground-truth lane points were 

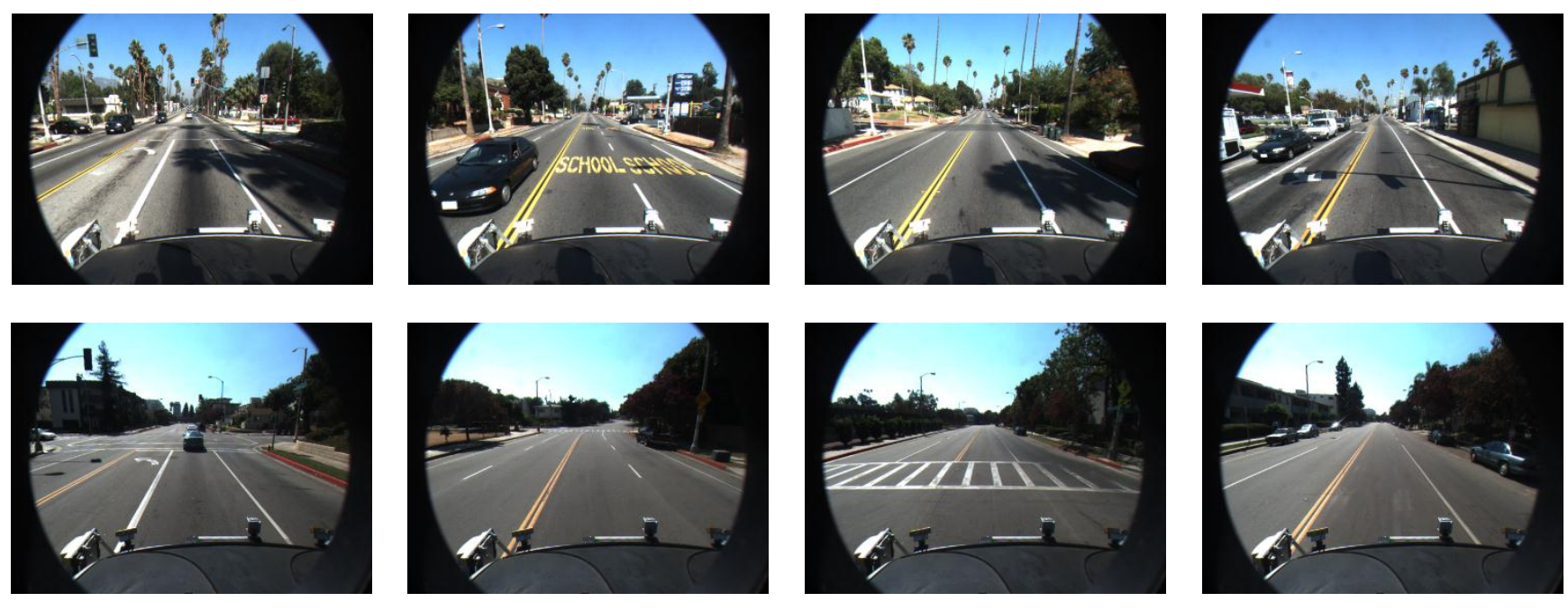

Fig. 7. Frames of the Caltech lanes dataset

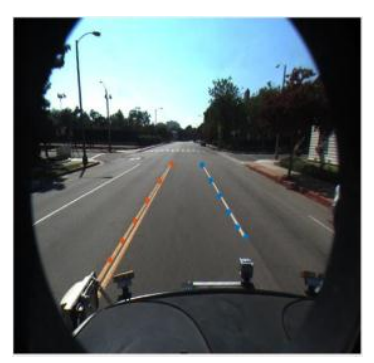

(a)

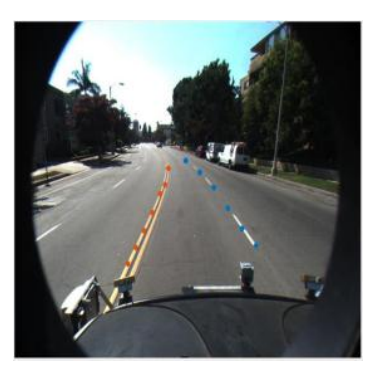

(b)
Fig. 8. ground-truth lane points.

expressed in image coordinates, yet estimated lane boundaries were modeled in the vehicle coordinates. Consequently, the ground-truth lane points were transformed from image coordinates to vehicle coordinates, and thus the estimated lane boundary models could be directly compared with them. Afterwards, we carried out the proposed method on each video sequence and calculated the estimated lane boundaries. We could then measure detection errors and evaluate the proposed method performance. So in order for an estimated model to be considered a match, i.e, true Positive, all the ground-truth lane points had to be within a specified lateral distance from the corresponding estimated lane boundary. This maximum lateral distance was set to 0.3 as per the quality requirements of lane detection systems. Otherwise, it was regarded as false positive.

The performance of the proposed method can be evaluated at a single-frame basis and globally on the entire video sequence. Inspecting the performance at the single-frame basis, helps in recognizing failure situations which can be bypassed by refining the proposed method. It was conducted via measuring the average lateral distance between the estimated lane boundaries and the ground-truth lane points. Fig. 10 depicts the frame time on the horizontal axis with the computed average lateral distance of right and left lane boundaries for each frame of the videos sequences, on the vertical axis. Conversely, the global analysis delivers an outline summary of correctly-detected lanes (true positives), incorrect detections (false postitves) and lanes that missed detection (false negatives). Regarding this approach, the proposed method was executed on each video sequence. The number of , $f p$ and $f n$ were subsequently calculated. Accordingly, the precision $(P)$, the recall $(R)$ and the $F 1$ score metrics could be computed as the following : 
Vol.41, No. 1. January 2022
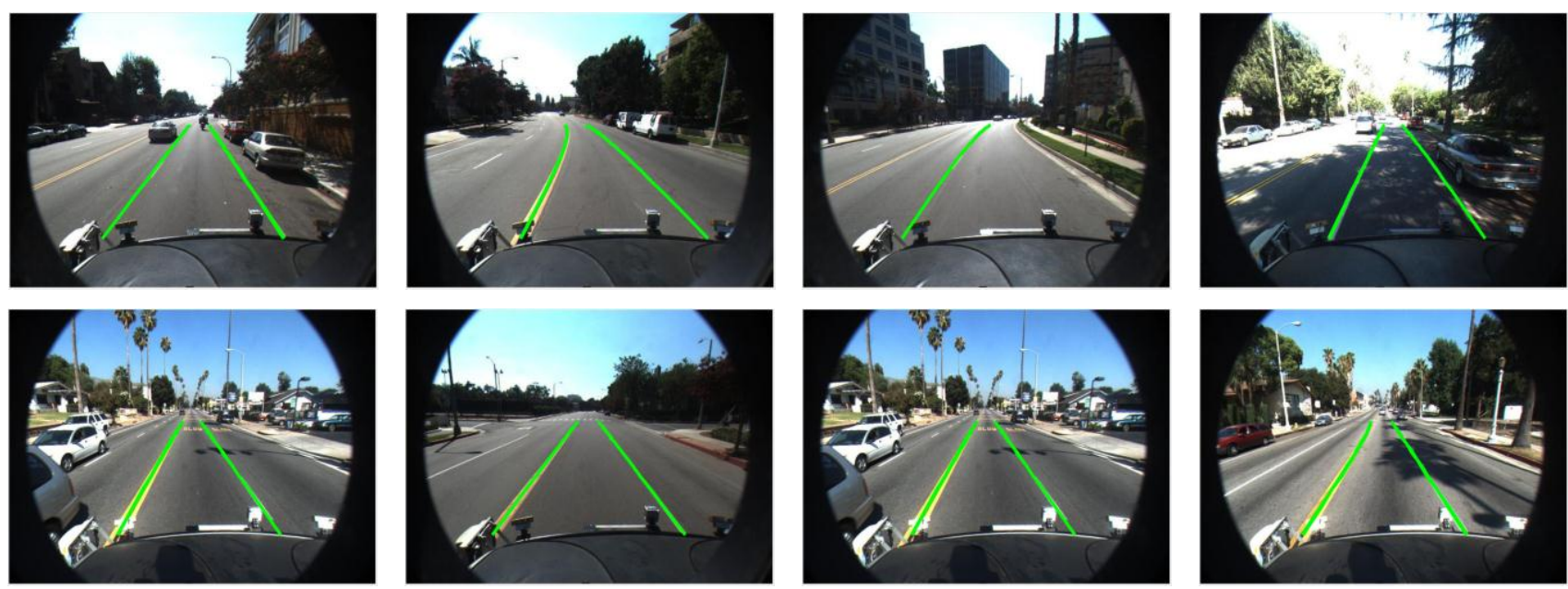

Fig. 9. Examples of correct detections in presence of different road structures, illumination variations, shadows, street writings.

$$
\begin{aligned}
P & =\frac{t p}{t p+f p} \\
R & =\frac{t p}{t p+f n} \\
F 1 & =\frac{2(P \cdot R)}{P+R}
\end{aligned}
$$

Table 1 depicts a summary of the performance evaluation of the proposed lane detection method using the Caltech dataset.

\section{B. Comparative analysis}

In this section, the detection performance of the quadratic model used in the proposed method is compared to other mathematical models that are commonly adopted in the literature such as the linear model $[20,21]$, and the cubic model [11]. Table 2 compares the $F 1$ scores measured using the performance evaluation criteria described earlier and obtained by the aforementioned models. In addition, the computational times per frame for each model are compared and summarized in Table 3. It shows the average time per frame computed for each video sequence using the different models and the corresponding overall average time per frame for each model.

\section{DISCUSSION}

As shown in Fig. 9, the detection results demonstrate the effectiveness of the proposed method in lane detection in diverse conditions in urban roads, including curved lanes, different road structures, illumination variations, shadows and presence of street writings.

Table 1. The performance evaluation of the proposed method

\begin{tabular}{|c|c|c|c|c|c|c|}
\hline Clip & tp & $\mathbf{f p}$ & $\mathbf{f n}$ & $\mathbf{P}$ & $\mathbf{R}$ & F1 \\
\hline C1 & 422 & 59 & 23 & 0.877 & 0.948 & 0.911 \\
\hline C2 & 391 & 311 & 65 & 0.557 & 0.857 & 0.675 \\
\hline W1 & 439 & 169 & 196 & 0.722 & 0.691 & 0.706 \\
\hline W2 & 352 & 104 & 92 & 0.772 & 0.793 & 0.782 \\
\hline
\end{tabular}

Table 2. The F1 score comparison of the linear, quadratic and cubic models

\begin{tabular}{|c|c|c|c|}
\hline Clip & Linear & Quadratic & Cubic \\
\hline
\end{tabular}


Vol.41, No. 1. January 2022

\begin{tabular}{|c|c|c|c|}
\hline $\mathrm{C} 1$ & 0.84934 & 0.92573 & 0.82162 \\
\hline $\mathrm{C} 2$ & 0.6893 & 0.67647 & 0.59805 \\
\hline $\mathrm{W} 1$ & 0.72626 & 0.70569 & 0.56551 \\
\hline $\mathrm{W} 2$ & 0.80222 & 0.80178 & 0.62838 \\
\hline
\end{tabular}

Table 3. The average time computed for each vide sequence in milliseconds per frame using the different models.

\begin{tabular}{|c|c|c|c|}
\hline Clip & Linear & Quadratic & Cubic \\
\hline C1 & 30.4062 & 31.6134 & 35.2556 \\
\hline C2 & 27.5333 & 28.5930 & 29.7369 \\
\hline W1 & 27.2293 & 29.4027 & 31.0078 \\
\hline W2 & 27.6405 & 32.8949 & 32.4529 \\
\hline Average & 28.2023 & 30.6260 & 32.1133 \\
\hline
\end{tabular}

Owing to the fact that the Caltech dataset has marked the ground-truth lane points of the double yellow lane boundaries on the outer boundary instead of the inner boundary or between them as can be seen in Fig. 8, the average lateral distances between the estimated lane boundaries and the corresponding ground-truth data of left lane boundaries tend to be quite greater than those of right lane boundaries as shown in Fig. 10.

The factor accounting for the relatively low performance of the proposed method on Cordova2 video compared to others in Table 1, is that the ego-vehicle was driving on the right lane of the road that had no right lane boundary most of the time, so the curb was detected as the right lane boundary as illustrated in Fig. 11. Yet, this doesn't really degrade the proposed method performance since the curb actually works as a lane boundary, even though it's not labeled as such in the Caltech dataset.

It can be seen from Table 2 that the linear and quadratic models outperform the cubic model in modeling lane boundaries in urban roads. At the same time, the cubic model comes with the highest average computational time per frame as illustrated in Table 3. As a result, it's not recommended for lanes modeling.

As shown in the the third column of Table 3 , the overall process of the proposed method requires only 30.63 milliseconds per frame, and thus it can be used for real-time applications. At the same time, it is faster and consumes less processing time than the real-time lane detection method presented in [8] which costs 33 milliseconds per frame.

In regards to the comparison of the detection performance and the computational time between the linear model and the quadratic one, it can be seen from Table 2 that both models achieve comparable performance in Cordova2, Washington1, Washington 2 video sequences where the roads are straight most of the time. However, the quadratic model surpasses the linear model in in the detection accuracy by $8.994 \%$ in Cordoval video sequence which exhibits curved lane boundaries and a wider variety of lane shapes. Moreover, this advantage doesn't come at the cost of computational time and complexity because the average computational time per frame calculated for the linear model and the quadratic oneare 
Vol.41, No. 1. January 2022

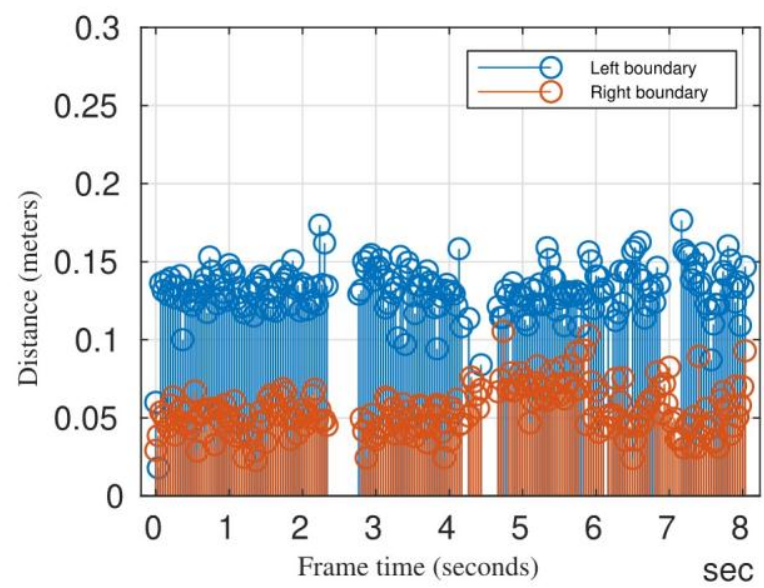

(a)

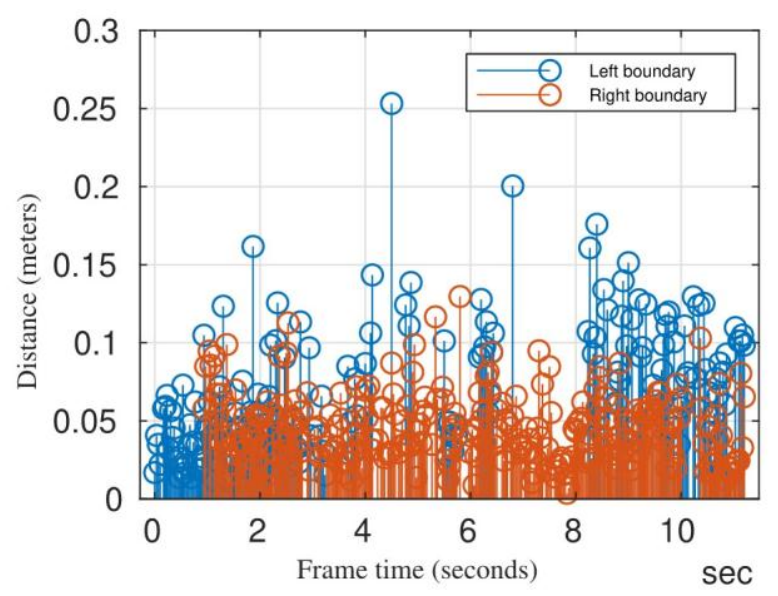

(c)

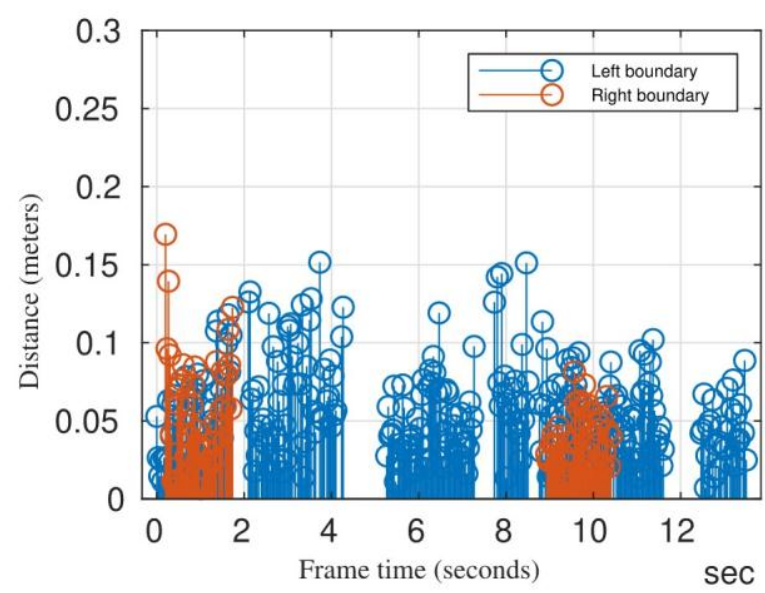

(b)

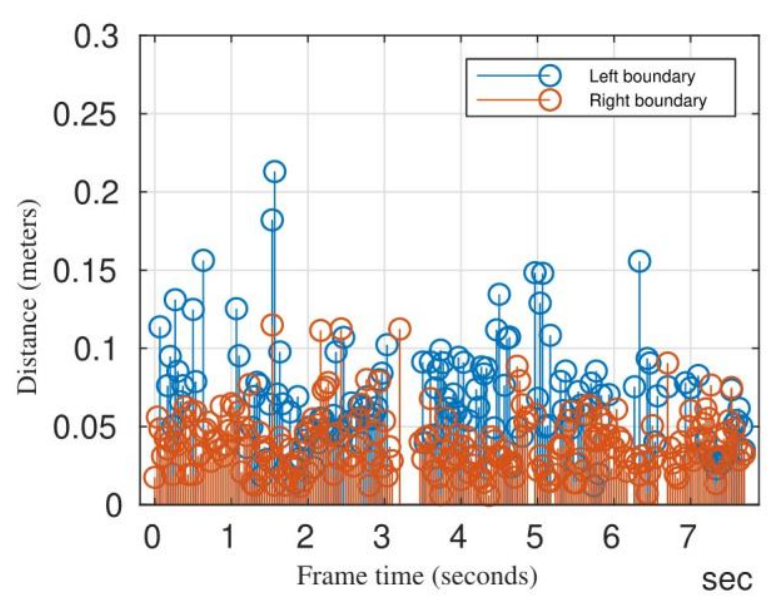

(d)

Fig. 10. Average lateral distance per frame. (a) Cordova1. (b) Cordova2. (c) Washington1. (d) Washington2

28.20 and 30.63 miiliseconds per frame, respectively.

In the proposed method, we employed the gray scale intensity image in the feature extraction sub method. However, there is still room for improvement since it leads to some incorrect detections, i.e, false positives and false negatives, in the case of bright yellow lane boundaries as depicted in Fig. 12. We intend to investigate different color representations that give the proposed method more robustness in detecting both white and yellow markings in more diverse illumination conditions. This will also allow us to improve the performance of the proposed method, specifi- cally in Washington 1 and Washington2 video sequences where complex situations are present.

\section{CONCLUSION}

A vision-based proposed method for lane detection, was presented in this paper. It specified a ROI to to focus on the part of the road relevant to the lane detection task. The IPM was subsequently applied to obtain a BEV of the 


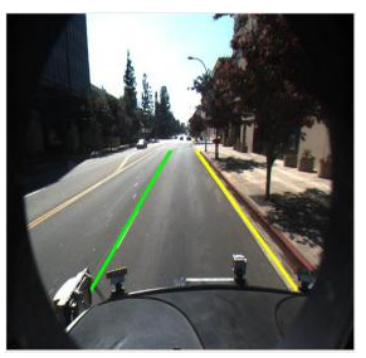

(a)

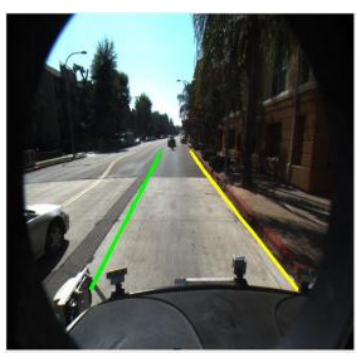

(b)
Fig. 11. Examples of false positive detections of Cordova2. True positives are represented in green, while False positives are represented in yellow.

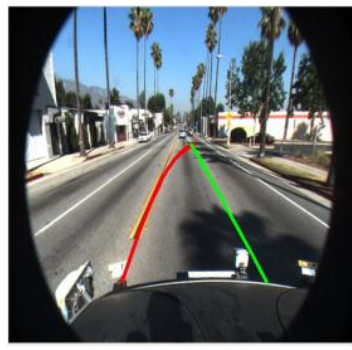

(a)

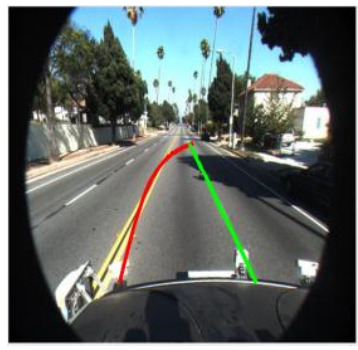

(b)
Fig. 12. Examples of incorrect detections. True positives are in green, but incorrect detections are represented in red.

road for the purpose of reducing the perspective distortion and simplifying the processes of lane features extraction and models fitting. Afterwards, the BEV image was converted into a black and white image where the white pixels represented the potential lane pixels through using the distinct colors of lane markings to achieve lane markings candidate detection. The RANSAC algorithm was then used to estimate the parameters of the quadratic lane models which were fitted to those points. Finally, the identified and estimated lane boundaries were overlaid on the original road image.

In order to check the accuracy of the proposed method, quantitative evaluation of the detection performance was conducted using the Caltech dataset. The detection performance and the computational time of the quadratic model used in the proposed method was measured and compared to other models adopted in the literature. The quadratic model surpassed the commonly used linear model in in the detection accuracy by $8.994 \%$ in Cordoval video sequence which exhibited curved lane boundaries and a wider variety of lane shapes. Moreover, this didn't come at the cost of the computational time because the average computational time per frame calculated for the quadratic model was 30.63 milliseconds per frame which makes it suitable for realtime applications.

The Detection results indicate that the proposed method demonstrates stable performance in different urban roads conditions and varying driving scenes, with room for further improvement through giving the proposed method more robustness in detecting yellow lane markings in more intense illumination conditions.

\section{REFERENCES}

1] World health organization, "Global status report on road safety 2018". Available: "https: //www.who.int/violence_injury_prevention/road_s afety_status/2018/en/”, accessed July 7, 2020.

[2] Association for safe international road travel, "Annual global road crash statistics 2020". Available: "https://www.asirt.org/safe-travel/roadsafety-facts/", accessed July 27, 2020.

[3] X. Luo, W. Du, and J. Zhang, "Safety benefits of belt pretensioning in conjunction with precrash braking in a frontal crash," in 2015 IEEE Intelligent Vehicles Symposium (IV), 2015, pp. 871-876: IEEE.

[4] J. Antony and S. Manikandan, "Expanding vision-based ADAS for non-structured envi- 
ronments," IET Intelligent Transport Systems, vol. 14, no. 6, pp. 620-627, 2020.

[5] W. Li, X. Gong, Y. Wang, and P. Liu, "A lane marking detection and tracking algorithm based on sub-regions," in Proceedings 2014 International Conference on Informative and Cybernetics for Computational Social Systems (ICCSS), 2014, pp. 68-73: IEEE.

[6] Y. Bian, J. Ding, M. Hu, Q. Xu, J. Wang, and K. Li, "An Advanced Lane-Keeping Assistance System With Switchable Assistance Modes," IEEE Transactions on Intelligent Transportation Systems, vol. 21, no. 1, pp. 385-396, 2019.

[7] J. Ruyi, K. Reinhard, V. Tobi, and W. Shigang, "Lane detection and tracking using a new lane model and distance transform," Machine vision and applications, vol. 22, no. 4, pp. 721-737, 2011.

[8] J. Son, H. Yoo, S. Kim, and K. Sohn, "Real-time illumination invariant lane detection for lane departure warning system," Expert Systems with Applications, vol. 42, no. 4, pp. 18161824, 2015.

[9] H. Kong, J.-Y. Audibert, and J. Ponce, "Vanishing point detection for road detection," in 2009 IEEE Conference on Computer Vision and Pattern Recognition, 2009, pp. 96-103: IEEE.

[10] A. S. Huang, D. Moore, M. Antone, E. Olson, and S. Teller, "Finding multiple lanes in urban road networks with vision and lidar," Autonomous Robots, vol. 26, no. 2-3, pp. 103-122, 2009.

[11] Z. Kim, "Robust lane detection and tracking in challenging scenarios," IEEE Transactions on Intelligent Transportation Systems, vol. 9, no. 1, pp. 16-26, 2008.

[12] M. Aly, "Real time detection of lane markers in urban streets," in 2008 IEEE Intelligent Vehicles Symposium, 2008, pp. 7-12: IEEE.
[13] C. Ma, L. Mao, Y. Zhang, and M. Xie, "Lane detection using heuristic search methods based on color clustering," in 2010 International Conference on Communications, Circuits and Systems (ICCCAS), 2010, pp. 368-372: IEEE.

[14] J. Huang, H. Liang, Z. Wang, Y. Song, and Y. Deng, "Lane marking detection based on adaptive threshold segmentation and road classification," in 2014 IEEE International Conference on Robotics and Biomimetics (ROBIO 2014), 2014, pp. 291-296: IEEE.

[15] F. You, R. Zhang, L. Zhong, H. Wang, and $\mathrm{J} . \mathrm{Xu}$, "Lane detection algorithm for nighttime digital image based on distribution feature of boundary pixels," Journal of the Optical Society of Korea, vol. 17, no. 2, pp. 188-199, 2013.

[16] G. Liu, S. Li, and W. Liu, "Lane detection algorithm based on local feature extraction," in 2013 Chinese Automation Congress, 2013, pp. 5964: IEEE.

[17] H. Yoo, U. Yang, and K. Sohn, "Gradientenhancing conversion for illumination-robust lane detection," IEEE Transactions on Intelligent Transportation Systems, vol. 14, no. 3, pp. 10831094, 2013.

[18] M. Nieto, J. A. Laborda, and L. Salgado, "Road environment modeling using robust perspective analysis and recursive Bayesian segmentation," Machine Vision and Applications, vol. 22, no. 6, pp. 927-945, 2011.

[19] M. A. Fischler and R. C. Bolles, "Random sample consensus: a paradigm for model fitting with applications to image analysis and automated cartography," Communications of the ACM, vol. 24, no. 6, pp. 381-395, 1981.

[20] A. Borkar, M. Hayes, and M. T. Smith, "Robust lane detection and tracking with ransac and kalman filter," in 2009 16th IEEE International Conference on Image Processing (ICIP), 2009, pp. 3261-3264: IEEE. 
Vol.41, No. 1. January 2022

[21] G. Zhang, N. Zheng, C. Cui, Y. Yan, and Z. Yuan, "An efficient road detection method in noisy urban environment," in 2009 IEEE Intelligent Vehicles Symposium, 2009, pp. 556-561: IEEE. 


\section{طريقة كفؤ للتعرف علي علامات الحارة المرورية في الطرق الحضرية}

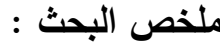

العديد من انظمة النقل الذكية مثل الأنظمة المتقدمة لمساعدة السائق يتم يتطويرها حاليا من اجل زيادة أمان الطرق و حركة المرور ـ تمتلك الأنظمة المتقدمة لمساعدة السائق القدرة علي إنقاذ الأرواح و تقليل عدد الحوادث علي الطرقات عن طريق تقليل الخطأ البشري أثناء عملية القيادة. أحد ثلاك الأنظمة هو نظام التعرف علي علامات حارة السير و التي ينت تصميمها من أجل التعرف علي علامات الحارة و تحديد اماكن تواجدها علي الطريق أمام السيارة التي يقودها السائق ، لذلك يعتبر هذا النظام مكون حاسم و أساسي تعنمد عليه أنظمة اخري متقدمة منل نظام إبقاء السيارة داخل حارةالسير المخصصة و .نظام تتبيه السائق عند مغادرة حارة السير . نقدم في هذه الورقة البحثنة طريقة مقترحة للتعرف علي علامات الحارة المرورية في الطرق الحضرية بإستخدام خوارزميات الروئة الحاسوبية. يتبعها تقييم متعمق للأداء بإستخدام مجموعة بيانات كالتنيك و مقارنة تحليلية بين الدالة التربعية التي استخدمت في الطريقة المقترحة و دوال أخري قدمت في دراسات أخري ـ نتائج التقبيم توضح فاعلية الطريقة المقترحة في التعرف علي علامات حارة السير في ظروف مختلفة للقيادة في الطرق الحضرية مثل الطرق المنحنية و وجود الظلال و التغييرات في ظروف الإضاءة و وجود العلامات الأرضية في الثوارع. تستغرق عملية المعالجة منوسط زمن 30.63 مللي ثانبة لكل إطار مما يجعلها مناسبة لنطبيقات الوقت الحقيقي. 